\title{
A NOTE ON GENERALIZED RESOLVENTS FOR ORDINARY DIFFERENTIAL OPERATORS
}

\author{
SUNG J. LEE
}

\begin{abstract}
We give an explicit construction for the kernel of an arbitrary generalized resolvent for an ordinary symmetric differential operator. In particular, this avoids the use of approximation of selfadjoint operators on compact intervals. We also discuss integrability of functions which are adjoint to certain fundamental solutions.
\end{abstract}

Coddington [2] showed that the kernel of an arbitrary generalized resolvent for a symmetric ordinary differential operator is the sum of two kernels: One is obtained by approximating selfadjoint operators on compact intervals and the other is obtained by considering the range of the resolvent (see [2, $(2.4)]$; also [1, p. 179]). In this paper we will give an explicit construction for the kernel of an arbitrary generalized resolvent as above. In particular, this avoids the use of approximation of selfadjoint operators. We also discuss integrability of functions which are adjoint to certain fundamental solutions.

Unexplained notation and terminology will be as in [2]. Let $L$ denote the formally selfadjoint differential operator $P_{0}(x) D^{n}+P_{1}(x) D^{n-1}+\cdots+$ $P_{n}(x)$ where $D=d / d x$, the $P_{k}$ are complex-valued functions of class $C^{n-k}$ on an open interval $(a, b)(-\infty \leqslant a<b \leqslant \infty)$ and $P_{0}(x) \neq 0$ on $(a, b)$. This operator $L$ generates a minimal symmetric operator $T_{0}$ and a maximal operator $T$ in the Hilbert space $\mathcal{H}=\mathscr{L}^{2}(a, b)$. Let $R(l)$ denote a generalized resolvent for $T_{0}$. Then $R$ is given by

$$
R(l)=\left(T_{A(l)}-l\right)^{-1}(9 l>0) ; \quad R(\bar{l})=(R(l))^{*} .
$$

THEOREM 1 (CODDINGTON [2]). The generalized resolvent $R(l)$ is an integral operator of Carleman type having a kernel $K=K(x, y, l)$ with the property that $\partial^{j+k-2} K / \partial x^{j-1} \partial y^{k-1}(j, k=1, \ldots, n)$ are continuous in $(x, y, l)$ and analytic in $l$ on any region for which $9 l \neq 0$, except for $x=y$ when $j$ or $k$ is $n$.

Our purpose here is to compute $K$ explicitly. Let $c$ be an arbitrary, but fixed point in $(a, b)$. Let $\alpha(l)$ denote the maximum number of linearly independent $\mathcal{L}^{2}(a, c)$-solutions of $L f=l f$. Let $\beta(l)$ denote the maximum number of linearly independent $\mathcal{L}^{2}(c, b)$-solutions of $L f=l f$. Finally let $\omega(l)$ denote the maximum number of linearly independent $\mathcal{L}^{2}(a, b)$-solutions of $L f=l f$. It is well known that $\alpha(l), \beta(l), \omega(l)$ only depend on whether $q l>0$

Received by the editors April 17, 1975 and, in revised form, August 20, 1975.

AMS (MOS) subject classifications (1970). Primary 34B25.

Key words and phrases. Symmetric differential operator, generalized resolvent, fundamental solutions.

(๑) American Mathematical Society 1976 
or $9 l<0$. When $9 l>0$, we put $\omega(l)=\omega^{+}, \omega(\bar{l})=\omega^{-}$. It is shown in [3] and [5] that $\alpha(l)+\beta(l)=\omega(l)+n$ for $g l \neq 0$. Using this equality and linear independence, we can choose fundamental solutions $\zeta_{1}(x, l), \ldots, \zeta_{n}(x, l)$ of $L f=l f$ $(\Im l \neq 0)$ such that $\left\{\zeta_{i}(x, l): 1 \leqslant i \leqslant \omega(l)\right\} \subset \mathcal{L}^{2}(a, b) ;\left\{\zeta_{i}(x, l): \omega(l)<i \leqslant \alpha(l)\right\}$ $\subset \mathfrak{L}^{2}(a, c) ;\left\{\zeta_{i}(x, l): \alpha(l)<i \leqslant n\right\} \subset \mathcal{L}^{2}(c, b)$ (cf. [8, pp. 90-91]). Let

$$
\zeta_{i *}(y, l)=(-1)^{n+i} W_{i}\left(\zeta_{1}, \ldots, \zeta_{n}\right)(y) / P_{0}(y) W\left(\zeta_{1}, \ldots, \zeta_{n}\right)(y)
$$

for $1 \leqslant i \leqslant n$. Here $W_{i}\left(\zeta_{1}, \ldots, \zeta_{n}\right)(y)$ denotes the determinant obtained from the wronskian $W\left(\zeta_{1}, \ldots, \zeta_{n}\right)(y)$ of $\zeta_{1}(y, l), \ldots, \zeta_{n}(y, l)$ by removing its $n$th row and $i$ th column. Let $\lambda$ be an arbitrary, but fixed complex number with $g \lambda>0$. For any $f$ in $\mathscr{L}^{2}(a, b)$ with compact support on $(a, b)$, set $u=R(\lambda) f$. Then $\left(T_{A(\lambda)}-\lambda\right) u=(L-\lambda) u=f$. Thus, using the variation of constants formula and the definitions of $\alpha(\lambda), \beta(\lambda)$ and $\omega(\lambda)$, we have

$$
R(\lambda) f=\sum_{1}^{\omega^{+}} \zeta_{i}(x, \lambda) a_{i}+\int_{a}^{b} \Omega_{0}(x, y, \lambda) f(y) d y
$$

for some complex constants $a_{i}$. Here for $9 l \neq 0$,

$$
\Omega_{0}(x, y, l)= \begin{cases}-\sum_{\omega(l)+1}^{\alpha(l)} \zeta_{i}(x, l) \zeta_{i *}(y, l), & x \leqslant y, \\ \sum_{1}^{\omega(l)} \zeta_{i}(x, l) \zeta_{i *}(y, l) & \\ +\sum_{\alpha(l)+1}^{n} \zeta_{i}(x, l) \zeta_{i *}(y, l), & x>y .\end{cases}
$$

Since $R(\lambda) f \in \mathscr{D}(\lambda)$, we have $\left\langle R(\lambda) f v_{j}^{*}(\lambda)\right\rangle=0$ for $j=1,2, \ldots, \omega^{+}$(see $[2$, p. 381]). In view of (1), this can be written

$$
\sum_{1}^{\omega^{+}} a_{i}\left\langle\zeta_{i}(\cdot, \lambda) v_{j}^{*}(\cdot, \lambda)\right\rangle=-\int_{a}^{b}\left\langle\Omega_{0}(\cdot, y, \lambda) v_{j}^{*}(\cdot, \lambda)\right\rangle f(y) d y
$$

for $j=1,2, \ldots, \omega^{+}$. Let $\Delta(\lambda)$ denote the $\omega^{+} \times \omega^{+}$matrix with $(j, i)$ entry $\left\langle\zeta_{i}(\cdot, \lambda) v_{j}^{*}(\cdot, \lambda)\right\rangle$. Clearly $\Delta(\lambda)$ is nonsingular. Let $\zeta^{+}(x, \lambda)$ denote the $1 \times \omega^{+}$ row vector $\left(\zeta_{i}(x, \lambda)\right)$ and let $\mathbf{V}_{*}(y, \lambda)$ denote the $\omega^{+} \times 1$ column vector $\left(v_{j}^{*}(y, \lambda)\right)$. Then substituting the solutions $\left(a_{i}\right)$ to $(2)$ into $(1)$, we get

$$
R(\lambda) f=\int_{a}^{b} \Omega(x, y, \lambda) f(y) d y
$$

where

$$
\Omega(x, y, \lambda)=\Omega_{0}(x, y, \lambda)-\zeta^{+}(x, \lambda) \Delta^{-1}(\lambda)\left\langle\Omega_{0}(\cdot, y, \lambda) \mathbf{V}_{*}(\cdot, \lambda)\right\rangle .
$$

Similarly, we have

$$
(R(\lambda))^{*} f=\int_{a}^{b} \Omega(x, y, \bar{\lambda}) f(y) d y
$$

where

$$
\Omega(x, y, \bar{\lambda})=\Omega_{0}(x, y, \bar{\lambda})-\zeta^{-}(x, \bar{\lambda}) \Delta^{-1}(\bar{\lambda})\left\langle\Omega_{0}(\cdot, y, \bar{\lambda}) \mathbf{V}(\cdot, \lambda)\right\rangle .
$$

Here $\zeta^{-}(x, \bar{\lambda})$ denotes the $1 \times \omega^{-}$row vector $\left(\zeta_{i}(x, \bar{\lambda})\right)$ and $\Delta(\bar{\lambda})$ denotes the $\omega^{-} \times \omega^{-}$nonsingular matrix with $(j, i)$ entry $\left\langle\zeta_{i}(\cdot, \bar{\lambda}) v_{j}(\cdot, \lambda)\right\rangle$. Finally $\mathbf{V}(x, \lambda)$ denotes the $\omega^{-} \times 1$ column vector $\left(v_{i}(x, \lambda)\right)$. Since $(R(\lambda))^{*}=R(\bar{\lambda})$; 
we see that $\Omega(x, y, \lambda)=\bar{\Omega}(y, x, \bar{\lambda})$. From this symmetry it can be shown easily that $\Omega(x, y, \lambda)$ belongs to $\mathcal{L}^{2}(a, b)$ as a function of $y$. Thus, since $R(\lambda)$ is a bounded operator, (3) holds for every $f$ in $\mathfrak{L}^{2}(a, b)$. Hence together with Theorem 1 we have $\int_{a}^{b}(K(x, y, \lambda)-\Omega(x, y, \lambda)) f(y) d y=0$ for any $f \in$ $\mathfrak{L}^{2}(a, b)$. This yields

THEOREM 2. $K(x, y, l)=\Omega(x, y, l)(q l \neq 0)$.

In view of $\Omega$ and using the definitions of $\alpha(l), \beta(l)$ and $\omega(l), \Omega$ can be written

$$
\Omega(x, y, l)=\Omega_{0}(x, y, l)+\sum_{1}^{\omega(l)} \zeta_{i}(x, l) \theta_{i}(y, l)
$$

for $9 l \neq 0$. Since $\Omega$ is symmetric, for $y<x$, we have

$$
\begin{aligned}
\sum_{1}^{\omega(l)} \zeta_{i}(x, l) & {\left[\zeta_{i *}(y, l)+\theta_{i}(y, l)\right]+\sum_{\alpha(l)+1}^{n} \zeta_{i}(x, l) \zeta_{i *}(y, l) } \\
= & -\sum_{\omega(l)+1}^{\alpha(l)} \bar{\zeta}_{i}(y, \bar{l}) \bar{\zeta}_{i *}(x, \bar{l})+\sum_{1}^{\omega(l)} \bar{\zeta}_{i}(y, \bar{l}) \bar{\theta}_{i}(x, \bar{l}) .
\end{aligned}
$$

Since the $\zeta_{i}(x, l)$ are linearly independent, we have

THEOREM 3. For $g l \neq 0$,

$$
\begin{gathered}
\left\{\zeta_{i *}(y, l): \alpha(l)<i \leqslant n\right\} \cup\left\{\zeta_{i *}(y, l)+\theta_{i}(y, l): 1 \leqslant i \leqslant \omega(l)\right\} \\
\subset 1 . s .\left\{\bar{\zeta}_{i}(y, \bar{l}): 1 \leqslant i \leqslant \alpha(\bar{l})\right\} \subset \mathcal{L}^{2}(a, c) . \\
\zeta_{i *}\{(y, l): \omega(l)<i \leqslant \alpha(l)\} \cup\left\{\theta_{i}(y, l): 1 \leqslant i \leqslant \omega(l)\right\} \\
\subset \text { 1.s. }\left\{\bar{\zeta}_{i}(y, \bar{l}): 1 \leqslant i \leqslant \omega(\bar{l}), \alpha(\bar{l})<i \leqslant n\right\} \subset \mathcal{L}_{2}(c, b) . \\
\left\{\theta_{i}(x, l) \mid 1 \leqslant i \leqslant \omega(\bar{l})\right\} \subset \text { l.s. }\left\{\bar{\zeta}_{j}(x, \bar{l}): 1 \leqslant j \leqslant n\right\} \cap \mathfrak{L}^{2}(c, b) .
\end{gathered}
$$

Here 1.s. denotes "the linear span of".

When the left endpoint $a$ is regular, $\theta_{i}(y, l) \in \mathfrak{L}_{2}(a, b)$ for $1 \leqslant i \leqslant \omega(l)$. Thus in view of (4) we have

COROLlary. If the left endpoint of $(a, b)$ is a regular point, for each $l$ with $9 l \neq 0$, the map $f m \rightarrow \int_{a}^{b} \Omega_{0}(x, y, l) f(y) d s$ is a bounded operator on $\mathcal{L}_{2}(a, b)$.

\section{REFERENCES}

1. E. A. Coddington, The spectral matrix and Green's function for singular self-adjoint boundary value problems, Canad. J. Math. 6 (1954), 169-185. MR 16, 39.

2. , Generalized resolutions of the identity for symmetric ordinary differential operators, Ann. of Math. (2) 68 (1958), 378-392. MR 21 \#2094.

3. T. Kimura and M. Takahasi, Sur les opérateurs différentiels ordinaires linéaires formellement autoadjoints. I, Funkcial. Ekvac. 7 (1965), 35-90. MR 32 \#1399.

4. S. J. Lee, Ordinary differential operators with complex coefficients, Doctoral Dissertation, McMaster University, Hamilton, Ont., 1972. 
5. S. J. Lee, On boundary conditions for ordinary linear differential operators, J. London Math. Soc. (2) 12 (1975).

6. , Formally self-adjoint systems of differential equations, J. Math. Anal. Appl. (to appear).

7. , Integrability of certain solutions of differential equations, Period. Math. Hungarica (3) 7 (1976).

8. M. A. Naimark, Linear differential operators, GITTL, Moscow, 1954; English transl., Part II, Ungar, New York, 1968. MR 16, 702; 41 \#485.

Department of Mathematics, Queen's University, Kingston, Ontario, Canada 